\title{
GROUP RING IDEMPOTENTS WITH MINIMAL TRACE
}

\author{
D. A. R. WALLACE
}

\begin{abstract}
Let $G$ be a group and let $K$ be a field of characteristic 0 . Let $e \neq 0$ be an idempotent of the group ring $K(G)$. It is shown that the trace of $e$ attains its lower bound if and only if the support of $e$ has a specific form.
\end{abstract}

1. Introduction. Let $G$ be a group, let $K$ be a field of characteristic 0 and let $K(G)$ be the group ring of $G$ over $K$. For $\alpha=\sum_{x \in G} \alpha_{x} x \in K(G)$, the support of $\alpha$, denoted by $\operatorname{Supp}(\alpha)$, is the finite set $\left\{x: \alpha_{x} \neq 0\right\}$ and the trace of $\alpha$, denoted by $\operatorname{tr}(\alpha)$, is the coefficient $\alpha_{1}$. Let $e \neq 0$ be an idempotent of $K(G)$ and suppose $|\operatorname{Supp}(e)|=n$. Using an inequality of D. S. Passman [3, Lemma 1.1.7], D. Farkas has shown that $\operatorname{tr}(e) \geq 1 / n[2$, Remark 2.6]. We here give a more direct derivation of this second inequality and show that equality holds if and only if $\operatorname{Supp}(e)$ is a subgroup and $e$ is a primitive central idempotent of $K(\operatorname{Supp}(e))$ corresponding to a linear character of $\operatorname{Supp}(e)$ (Theorem 2.5).

2. Results. Throughout we follow the notation of $[3]$ and assume, without loss, that $K$ is a subfield of the complex numbers.

We begin with a theorem that characterizes for a finite group $S$ the idempotent of the type we wish to consider for $G$ an infinite group.

THEOREM 2.1. Let $S$ be a finite group of order $n$. Let $e \neq 0$ be an idempotent of $K(S)$. Then $\operatorname{tr}(e)=1 / n$ if and only if $e$ is the primitive, central idempotent associated with a linear character $\lambda: S \rightarrow K$, namely

$$
e=\frac{1}{n} \sum_{x \in S} \overline{\lambda(x)} x .
$$

ProOF. (i) Let $\lambda: S \rightarrow K$ be a linear character and let

$$
e=\frac{1}{n} \sum_{x \in S} \overline{\lambda(x)} x
$$

Then $e$ is the primitive, central idempotent associated with $\lambda[\mathbf{1}$, Lemma IV 7.1] and $\operatorname{tr}(e)=1 / n$.

(ii) Suppose $\operatorname{tr}(e)=1 / n$. By [2, Lemma 2.1.2(ii)]

$$
\operatorname{tr}(e)=\frac{\operatorname{dim} e K(S)}{n} .
$$

Thus $\operatorname{dim} e K(S)=1$ and so $e K(S)$ is an irreducible $K(S)$-module. Let $\lambda: S \rightarrow K$ be the linear character corresponding to the module $e K(S)$. Let $e=\sum_{x \in S} e_{x} x$. Then for $y \in S$

$$
e y^{-1}=\lambda\left(y^{-1}\right) e,
$$

Received by the editors February 27, 1986 .

1980 Mathematics Subject Classification (1985 Revision). Primary 16A27; Secondary 20 C07.

Key words and phrases. Group ring, idempotent, trace. 
which implies that

$$
\left(\sum_{x \in S} e_{x} x\right) y^{-1}=\lambda\left(y^{-1}\right)\left(\sum_{x \in S} e_{x} x\right) .
$$

Comparing the coefficient of 1 on both sides we have

$$
e_{y}=\lambda\left(y^{-1}\right) e_{1}=\overline{\lambda(y)} 1 / n \quad(y \in S),
$$

from which we obtain the result.

We now prove an elementary lemma from which we shall derive the aforementioned inequality, namely, $\operatorname{tr}(e) \geq 1 / n$.

LEMMA 2.2. Let $\lambda_{i} \in \mathbf{R}(i=1,2, \ldots, n)$. Then

$$
n\left(\sum_{i=1}^{n} \lambda_{i}^{2}\right)-\left(\sum_{i=1}^{n} \lambda_{i}\right)^{2} \geq 0
$$

with equality if and only if $\lambda_{1}=\lambda_{2}=\cdots=\lambda_{n}$.

PROOF.

$$
\begin{aligned}
n\left(\sum_{i=1}^{n} \lambda_{i}^{2}\right)-\left(\sum_{i=1}^{n} \lambda_{i}\right)^{2} & =(n-1)\left(\sum_{i=1}^{n} \lambda_{i}^{2}\right)-2\left[\sum_{\substack{i, j \\
i<j}}^{n} \lambda_{i} \lambda_{j}\right] \\
& =\sum_{\substack{i, j \\
i<j}}\left(\lambda_{i}-\lambda_{j}\right)^{2}
\end{aligned}
$$

from which the result follows.

Corollary 2.3. Let $0 \neq \alpha=\sum_{x \in G} \alpha_{x} x \in K(G)$. Then

$$
\frac{\|\alpha\|^{2}}{|\alpha|^{2}} \geq \frac{1}{|\operatorname{Supp}(\alpha)|}
$$

with equality if and only if $\left|\alpha_{x}\right|=\left|\alpha_{y}\right|$ for all $x, y \in \operatorname{Supp}(\alpha)$.

Proof. Let $S=\operatorname{Supp}(\alpha)$ and let $n=|\operatorname{Supp}(e)|$. Then by definition

$$
\|\alpha\|^{2}=\sum_{x \in S}\left|\alpha_{x}\right|^{2}, \quad|\alpha|=\sum_{x \in S}\left|\alpha_{x}\right|
$$

so

$$
n\|\alpha\|^{2}-|\alpha|^{2}=n\left[\sum_{x \in S}\left|\alpha_{x}\right|^{2}\right]-\left[\sum_{x \in S}\left|\alpha_{x}\right|\right]^{2} .
$$

Application of Lemma 2.2 yields the result.

Corollary 2.4 [2, Remark 2.6]. Let $0 \neq e=e^{2} \in K(G)$. Then

$$
\operatorname{tr}(e) \geq \frac{\|e\|^{2}}{|e|^{2}} \geq \frac{1}{|\operatorname{Supp}(e)|} .
$$

PROOF. This now follows immediately from Lemma 2.1.7 of [3].

We now come to the main theorem of this paper. 
THEOREM 2.5. Let $e \neq 0$ be an idempotent of $K(G)$ such that

$$
\operatorname{tr}(e)=1 / n
$$

where $n=|\operatorname{Supp}(e)|$. Then $S=\operatorname{Supp}(e)$ is a finite subgroup of $G$ and there exists a linear character $\lambda: S \rightarrow K$ such that

$$
e=\frac{1}{n} \sum_{x \in S} \overline{\lambda(x)} x
$$

PROOF. By Corollary 2.5

$$
\frac{\|e\|^{2}}{|e|^{2}}=\frac{1}{|\operatorname{Supp}(e)|}=\frac{1}{n} .
$$

Thus, letting $e=\sum_{x \in G} e_{x} x$, we have, by Corollary 2.3,

$$
\left|e_{x}\right|=\left|e_{1}\right|=e_{1}=1 / n \quad(x \in S) .
$$

Let $y \in S$. Then, as $e^{2}=e$, we have

$$
e_{y}=\sum_{\substack{x, z \\ x z=y}} e_{x} e_{z}=\sum_{x \in G} e_{x} e_{x^{-1}} .
$$

Now for $x \in S,\left|e_{x}\right|=1 / n$ and so

Thus

$$
\left|e_{x^{-1} y}\right|= \begin{cases}1 / n & \left(x^{-1} y \in S\right), \\ 0 & \left(x^{-1} y \notin S\right) .\end{cases}
$$

$$
\begin{aligned}
\frac{1}{n} & =\left|e_{y}\right| \leq \sum_{x \in G}\left|e_{x}\right|\left|e_{x^{-1} y}\right| \\
& =\sum_{x \in S}\left|e_{x}\right|\left|e_{x^{-1} y}\right| \leq \frac{1}{n} \sum_{x \in S}\left|e_{x^{-1} y}\right| .
\end{aligned}
$$

This is only possible if for all $x \in S\left|e_{x^{-1} y}\right|=1 / n$ or, equivalently, $x^{-1} y \in S$. But $y$ is arbitrary and so for all $x, y \in S, x^{-1} y \in S$ and hence $S$ is a subgroup. The remaining assertions follow from Theorem 2.1.

REMARK 2.6. The conclusion above does not hold if we merely suppose that $e$ is an idempotent for which

$$
\frac{\|e\|^{2}}{|e|^{2}}=\frac{1}{|\operatorname{Supp}(e)|} .
$$

To see this let $G$ be either the group $\left\langle x, y: y^{-1} x y=x^{-1}, y^{2}=1\right\rangle$, which is the infinite dihedral group, or its homomorphic image $\left\langle x, y: y^{-1} x y=x^{-1}, y^{2}=1\right.$, $\left.x^{3}=1\right\rangle$, which is the symmetric group of order 6 . Then

$$
e=\frac{\left(1-x+x^{-1}\right)+\left(x-x^{-1}-1\right) y}{2}
$$

is a noncentral idempotent in $\mathbf{Q}(G), \operatorname{tr}(e)=\frac{1}{2},|e|=3,\|e\|^{2}=\frac{3}{2}$ and

$$
\frac{\|e\|^{2}}{|e|^{2}}=\frac{1}{6}=\frac{1}{|\operatorname{Supp}(e)|} \text {. }
$$

In either case $G$ is generated by $\operatorname{Supp}(e)$ and in the second case $G=\operatorname{Supp}(e)$.

From our main theorem we obtain the following result which may be of independent interest. 
THEOREM 2.7. Let $0 \neq e=e^{2} \in K(G)$. Then

(i) $|e| \geq 1$, and

(ii) $|e|=1$ if and only if $S=\operatorname{Supp}(e)$ is a finite subgroup of $G$ of order $n$ and there exists a linear character $\lambda: S \rightarrow K$ such that

$$
e=\frac{1}{n} \sum_{x \in S} \overline{\lambda(x)} x .
$$

ProOF. By [3, Lemma 1.1.5(iii)] we have

$$
\|e\|=\|e e\| \leq\|e\||e|
$$

and so $|e| \geq 1$.

(ii) We remark that if $S=\operatorname{Supp}(e)$ is a finite subgroup of order $n$ and $e$ has the given form then it is an easy calculation that $|e|=1$.

Suppose, conversely, that $|e|=1$. Choose $m \in S$ such that

$$
\left|e_{m}\right|=\operatorname{Max}\left\{\left|e_{x}\right|: x \in S\right\} \text {. }
$$

Then, as $e^{2}=e$, we have $e_{m}=\sum_{u \in G} e_{u} e_{u^{-1} m}$ and hence

$$
\begin{aligned}
\left|e_{m}\right| & \leq \sum_{u \in G}\left|e_{u}\right|\left|e_{u^{-1} m}\right| \\
& =\sum_{u \in S}\left|e_{u}\right|\left|e_{u^{-1} m}\right| \leq \sum_{u \in S}\left|e_{m}\right|\left|e_{u^{-1} m}\right| \\
& \leq\left|e_{m}\right| \sum_{u \in S}\left|e_{u^{-1} m}\right| \leq\left|e_{m}\right||e| \\
& =\left|e_{m}\right| .
\end{aligned}
$$

Thus we have equality throughout and so, in particular, for all $u \in S$

$$
\left|e_{u}\right|=\left|e_{m}\right| \text {. }
$$

Thus $\left|e_{u}\right|=e_{1}(u \in S)$ and hence

$$
1=|e|=\sum_{x \in S}\left|e_{x}\right|=n e_{1} .
$$

'This yields $e_{1}=1 / n$ and the result now follows from Theorem 2.5.

ACKNOWLEDGMENT. This note was written while the author was visiting the University of Wisconsin-Madison, to whose Mathematics Department and to Don Passman in particular he would wish to express his thanks for kind and stimulating hospitality.

\section{REFERENCES}

1. W. Feit, The representation theory of finite groups, North-Holland, New York, 1982.

2. Z. Marciniak, Idempotents in group rings, Math Z. 184 (1983), 19-27.

3. D. S. Passman, Algebraic structure of group rings, Wiley-Interscience, New York, 1977.

DEPARTMENT OF MATHEMATICS, UNIVERSiTy OF WisCoNSIN-MADISON, VAN VLECK HALL, 480 LINCOLN DRIVE, MADISON, WISCONSIN 53706

Current address: Department of Mathematics, University of Strathclyde, Livingstone Tower, 26 Richmond Street, Glasgow, G1 1XH, Scotland 